EPJ Web of Conferences 52, 01010 (2013)

DOI: 10.1051/epjconf/20135201010

(C) Owned by the authors, published by EDP Sciences, 2013

\title{
New results from the NA49 experiment on hadron production in $p+p$ and $p+C$ interactions and survey of backward hadrons in $\mathrm{p}+\mathrm{C}$ collisions
}

\author{
M. Makariev ${ }^{1, a}$ for the NA49 collaboration \\ ${ }^{1}$ Institute for Nuclear Research and Nuclear Energy, Bulgarian Academy of Sciences, Sofia, Bulgaria
}

\begin{abstract}
Recent results on proton, anti-proton, neutron and charged kaon production in proton-proton and proton, anti-proton, neutron, deuteron and triton production in proton-carbon collisions at $158 \mathrm{GeV} / \mathrm{c}$ beam momentum are presented. Data samples of 4.8 million and 385734 inelastic events in $\mathrm{p}+\mathrm{p}$ and $\mathrm{p}+\mathrm{C}$, respectively, are obtained with the NA49 detector at the CERN SPS accelerator. The charged particles are identified by energy loss measurement in a system of four TPC chambers, while the neutrons are detected in a forward hadronic calorimeter. The data cover a major fraction of the phase space, ranging from 0 to $1.9 \mathrm{GeV}$ in $p_{T}$ and in Feynman $x$ variable from -0.8 to 0.95 for protons, from -0.2 to 0.3 for anti-protons, from 0.1 to 0.95 for neutrons and from 0 to 0.5 for kaons. The comparison of the results on proton and neutron production in $\mathrm{p}+\mathrm{p}$ interactions and deep inelastic $\mathrm{e}+\mathrm{p}$ collisions at HERA reveals an independence of target fragmentation on the projectile type. Using the charged kaon data in $p+p$ collisions as a reference, a new evaluation of the energy dependence of kaon production, including neutral kaons, is conducted over a range from $3 \mathrm{GeV}$ to $\mathrm{p}+\overline{\mathrm{p}}$ collider energies. A survey of backward production of protons and pions in $\mathrm{p}+\mathrm{C}$ collisions in the range of lab angles from 10 to 180 degrees, from 0.2 to $1.2 \mathrm{GeV} / \mathrm{c}$ in lab momentum and from 1 to $400 \mathrm{GeV} / \mathrm{c}$ in projectile momentum has been performed.
\end{abstract}

\section{Introduction}

In the absence of quantitative prediction in the nonperturbative sector of QCD, the study of soft hadronic physics has to rely on self-consistent high statistics data sets, including a variety of projectile and target combinations. In addition full phase space coverage with complete particle identification and small systematic uncertainty is required.

The NA49 experiment aims at providing such data sets ranging from elementary hadron-proton collisions to hadron-nucleus and nucleus-nucleus interactions, obtained using the same detector layout combining wide acceptance, systematic uncertainty of less than $5 \%$ and complete particle identification via measurement of specific energy loss. It is therefore well suited for the comparison of the different processes and to a detailed scrutiny of the evolution from elementary to nuclear hadronic phenomena and thus providing a basis for a model independent study to the underlying production mechanisms.

In the framework of this extensive experimental program the NA49 experiment has already published papers concerning pion [1], baryon [2] and kaon [3] production in $\mathrm{p}+\mathrm{p}$ collisions, and pion $[4,5]$ production in $\mathrm{p}+\mathrm{C}$ collisions. Two new articles have been submitted for publication regarding baryon and light ion production [6] and a survey of backward production of protons and pions from 1 to $400 \mathrm{GeV} / \mathrm{c}$ beam momentum [7] in $\mathrm{p}+\mathrm{C}$ interactions.
In this paper the recent results from [2, 3, 6, 7] will be discussed.

\section{Data sets}

The data sets obtained by the NA49 experiment are summarized in Fig. 1.

Table 1. The data sets obtained by the NA49 experiment.

\begin{tabular}{ccc}
\hline hadron-proton & hadron-nucleus & nucleus-nucleus \\
$\mathrm{p}+\mathrm{p}$ & $\mathrm{d}+\mathrm{p}$ & $\mathrm{Pb}+\mathrm{Pb}$ \\
$\mathrm{n}+\mathrm{p}$ & $\mathrm{p}+\mathrm{C}$ & \\
$\pi^{+}+\mathrm{p}$ & $\mathrm{p}+\mathrm{Pb}$ & \\
$\pi^{-}+\mathrm{p}$ & $\pi^{+}+\mathrm{Pb}$ & \\
& $\pi^{-}+\mathrm{Pb}$ & \\
\hline
\end{tabular}

\section{Variables and cross section definition}

The NA49 experiment establishes the invariant double differential cross sections

$$
f\left(x_{F}, p_{T}\right)=E\left(x_{F}, p_{T}\right) \cdot \frac{d^{3} \sigma}{d p^{3}}\left(x_{F}, p_{T}\right) .
$$

Here $p_{T}$ is the transverse momentum and $x_{F}$ is the reduced longitudinal momentum:

$$
x_{F}=\frac{p_{L}}{\sqrt{s} / 2}
$$

a e-mail: makariev@inrne.bas.bg 
where $p_{L}$ denotes the longitudinal momentum component in the center-of-mass system (cms). For the neutrons, due to the lack of transversal granularity of the NA49 calorimeter, only $p_{T}$-integrated density distributions:

$$
d n / d x_{F}=\pi / \sigma_{\text {inel }} \cdot \sqrt{s} / 2 \cdot \int f / E \cdot d p_{T}^{2}
$$

will be presented, with $\sigma_{\text {inel }}$ being total inelastic cross section.

\section{Results from $p+p$ interactions}

\subsection{Baryons}

It total 333 cross sections for protons and 143 for antiprotons have been derived, with systematic errors of $2.5 \%$ for protons and $3.3 \%$ for anti-protons, respectively, see [2]. The data cover the range in $x_{F}$ from -0.05 to $0.95(-0.05$ to 0.4$)$ and in $p_{T}$ from 0 to $1.9 \mathrm{GeV} / \mathrm{c}(0$ to $1.5 \mathrm{GeV} / \mathrm{c})$ for protons (anti-protons). An example of $x_{F}$ distributions of protons at fixed $p_{T}$ is shown in Fig. 1.

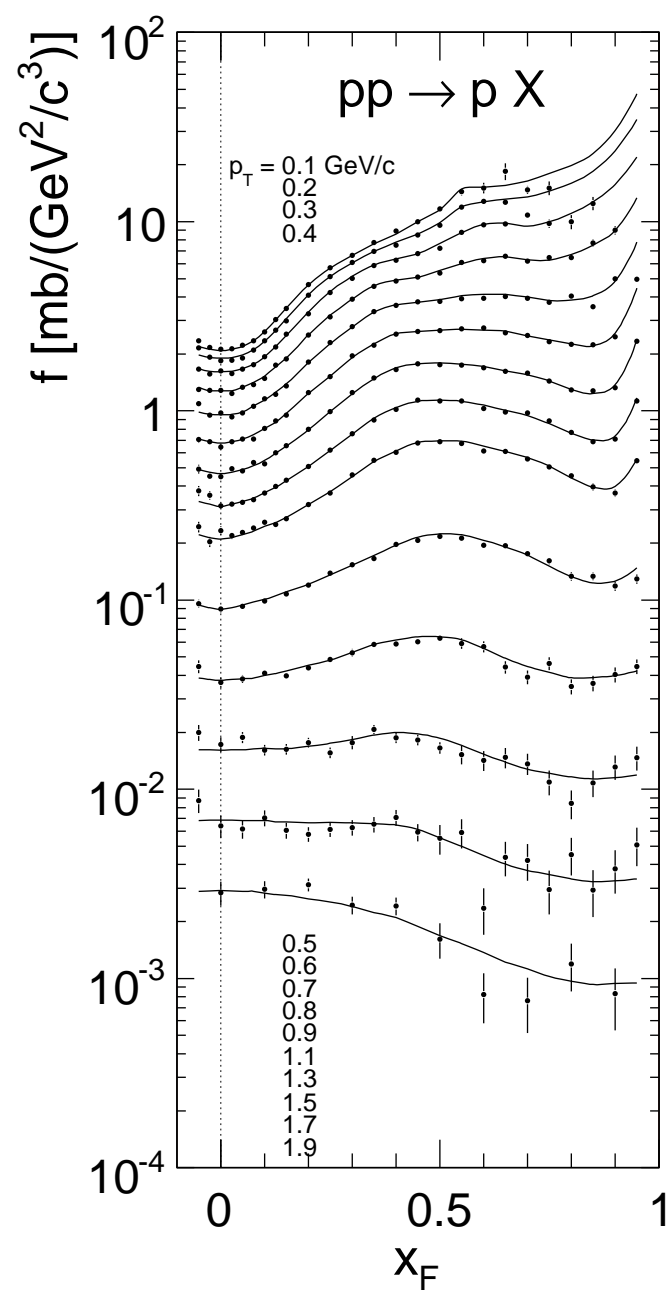

Figure 1. Double differential invariant cross section $f\left(x_{F}, p_{T}\right)$ $\left[\mathrm{mb} /\left(\mathrm{GeV}^{2} / \mathrm{c}^{3}\right)\right]$ as a function of $x_{F}$ at fixed $p_{T}$ for protons produced in $\mathrm{p}+\mathrm{p}$ collisions at $158 \mathrm{GeV} / \mathrm{c}$ beam momentum. The lines show the result of the interpolation of the data.
As the NA49 detector acceptance is limited at low $p_{T}$ and large $x_{F}$ the results from other measurements have been used to supplement the NA49 data, for a detailed discussion see [2]. The dense coverage allows for precise determination of integrated quantities which are also available in [2].

The $p_{T}$ integrated density distribution $d n / d x_{F}$ for neutrons is presented in Fig. 2 as a function of $x_{F}$. The $\mathrm{K}_{L}^{0}$ and $\overline{\mathrm{n}}$ distributions, which have been subtracted from the total measured yields, are also shown in the figure.

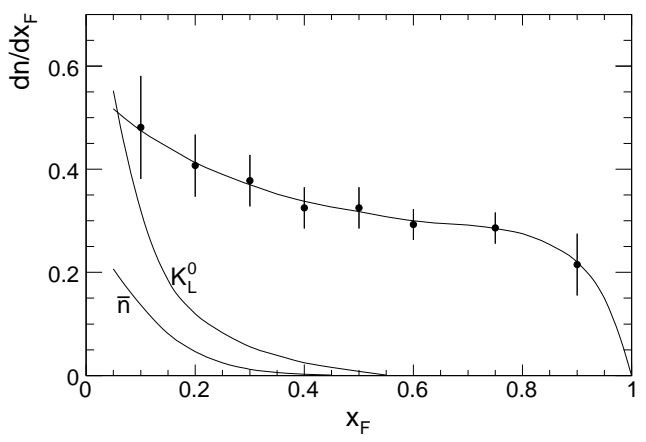

Figure 2. $p_{T}$ integrated density distribution $d n / d x_{F}$ as a function of $x_{F}$ of neutrons produced in $\mathrm{p}+\mathrm{p}$ interactions at $158 \mathrm{GeV} / \mathrm{c}$. The subtracted $\mathrm{K}_{L}^{0}$ and anti-neutron distributions are also shown.

\subsection{Comparison to $e+p$ collisions at HERA}

Data from the ZEUS collaboration at HERA concerning proton [8] and neutron [9] production in the target hemisphere provide results at mean energies of about $130 \mathrm{GeV}$ in the photon-proton cms. These data allow for a rather detailed comparison of the $\mathrm{e}+\mathrm{p}$ and $\mathrm{p}+\mathrm{p}$ interactions.

The ZEUS data cover a range from $0.1-0.7 \mathrm{GeV} / \mathrm{c}$ in $p_{T}$ and from 0.6 to 0.99 in $x_{F}$ for protons [8], and from 0.05 to $0.6 \mathrm{GeV} / \mathrm{c}$ in $p_{T}$ and from 0.26 to 0.97 in $x_{F}$ for neutrons [9]. The comparison of the shape of the $p_{T}$ distributions with the proton results from NA49 (full lines), normalized to the ZEUS data, is shown in Figs. 3 and 4.

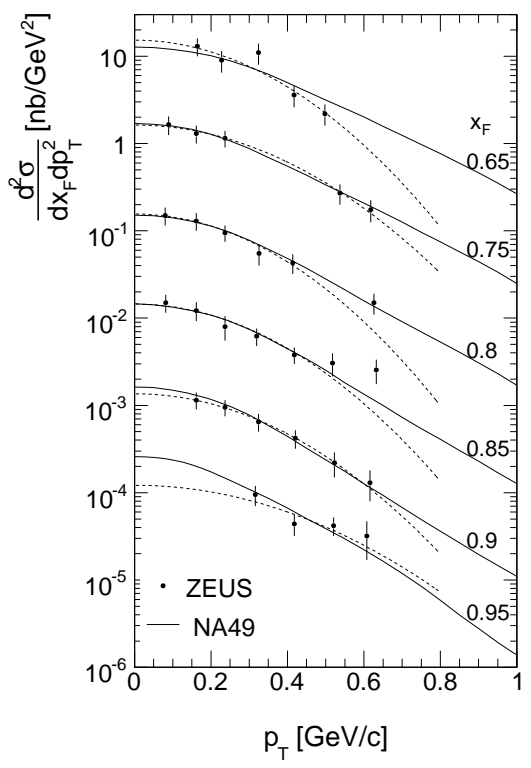

Figure 3. Comparison of the proton $p_{T}$ distributions at several $x_{F}$ values of the NA49 results (full lines) with measurements from [8]. The data were successively divided by 10 for different $x_{F}$ values for better separation. The dashed lines represent the parametrization used in [8]. 


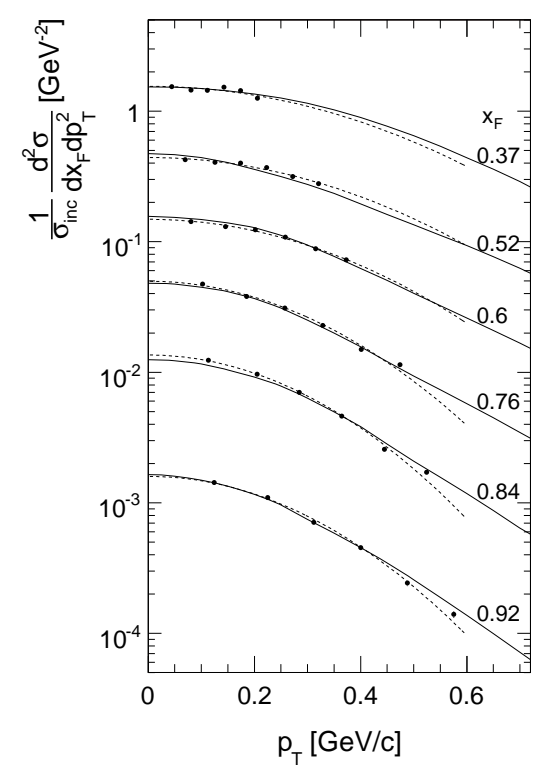

Figure 4. Comparison of the $p_{T}$ distributions at different $x_{F}$ values of protons from NA49 (full lines) with neutrons from [9]. The data were successively divided by 3 for different $x_{F}$ values for better separation. The dashed lines represent the parametrization used in [9].

This comparison reveals that the shape of the $p_{T}$ distributions is similar between the protons and neutrons as well as between the different interactions. Also the absolute proton and neutron densities have been shown to be equal in $\mathrm{e}+\mathrm{p}$ and $\mathrm{p}+\mathrm{p}$ collisions [2], corresponding to hadronic factorization.

\subsection{Kaons}

The results on kaon production consist of 158 data points for each $\mathrm{K}^{+}$and $\mathrm{K}^{-}$. They cover a range from 0 to $1.7 \mathrm{GeV} / \mathrm{c}$ in $p_{T}$ and from 0 to $0.4(0.5)$ in $x_{F}$ for $\mathrm{K}^{+}\left(\mathrm{K}^{-}\right)$ with a systematic uncertainty of $2.2 \%$ at $x_{F}<0.25$ and up to $10 \%$ for $\mathrm{K}^{+}$and $6 \%$ for $\mathrm{K}^{-}$at larger $x_{F}$. As in the case of baryons the integrated quantities have been established with high precision.

As the interaction energy of $\sqrt{s}=17.2 \mathrm{GeV}$ is located at a strategical point between threshold-dominated and scaling phenomena at lower and higher energies, respectively, a new and complete study of the $s$-dependence of kaon production including $\mathrm{K}_{S}^{0}$ has been attempted using the new NA49 data as a reference. This study covers the energy range $3<\sqrt{s}<1800 \mathrm{GeV}$ and aims at establishing an internally consistent picture of kaon production. The $\sqrt{s}$ dependence of the total kaon yields $\left\langle n_{\mathrm{K}}\right\rangle$ is presented in Fig. 5.

This new evaluation should replace the data from [10] which show an unphysical behaviour of charged kaon yield [3]. These results also prove the validity of the relation $\mathrm{K}_{S}^{0}=0.5\left(\mathrm{~K}^{+}+\mathrm{K}^{-}\right)$for $\sqrt{s}>5 \mathrm{GeV}$.

\section{Results from $p+C$ interactions}

\subsection{Baryons}

The data from $\mathrm{p}+\mathrm{C}$ collisions cover a phase space area ranging from 0 to $1.9 \mathrm{GeV} / \mathrm{c}$ in transverse momentum and from -0.80 to 0.95 for protons and from -0.2 to 0.3 for anti-protons in $x_{F}$ [6]. This results in 491 and 121 cross

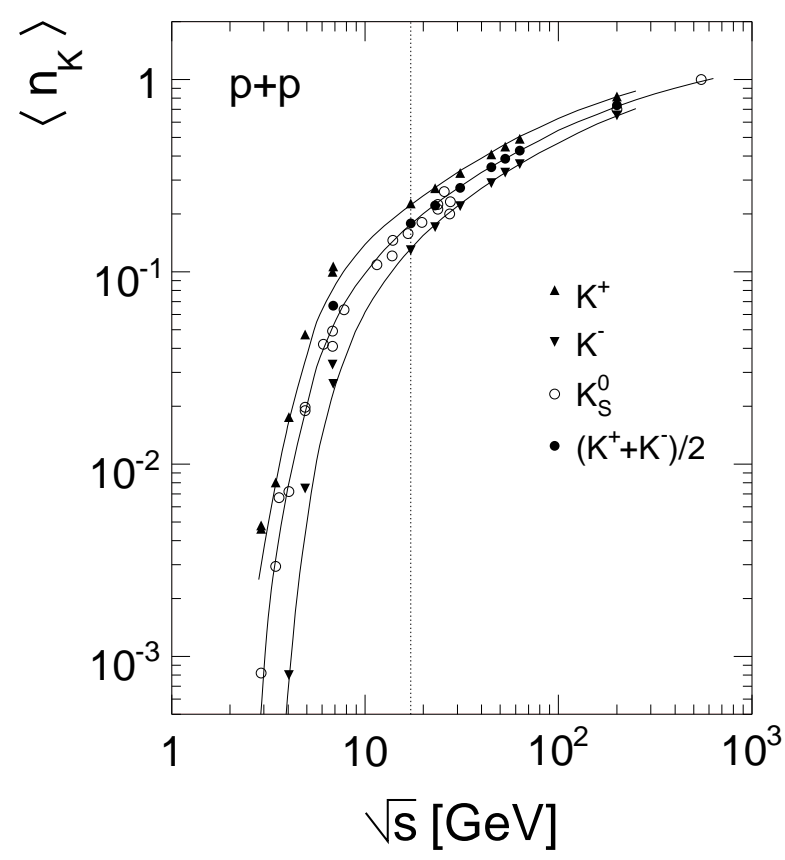

Figure 5. Total yields $\left\langle n_{\mathrm{K}^{+}}\right\rangle,\left\langle n_{\mathrm{K}^{-}}\right\rangle$and $\left\langle n_{\mathrm{K}_{S}^{0}}\right\rangle$ as a function of $\sqrt{s}$. The full line through the $\mathrm{K}_{S}^{0}$ is the result of an eyeball fit, the lines through the $\mathrm{K}^{+}$and $\mathrm{K}^{-}$data are derived from [3]. The full circles in the $\mathrm{K}_{S}^{0}$ data correspond to $0.5\left(\left\langle n_{\mathrm{K}^{+}}\right\rangle+\left\langle n_{\mathrm{K}^{-}}\right\rangle\right)$established at the corresponding $\sqrt{s}$ values.

section values for protons and anti-protons, respectively. Existing data [11], which are complementary to the NA49 measurements, in the far backward hemisphere are used to extend the coverage for protons into the region of intranuclear cascading, see [6]. The corresponding proton $x_{F}$ distributions, which cover the phase space from $x_{F}=-2$ up to the kinematic limit at $x_{F} \sim+1$, are shown in Fig. 6 . Both the NA49 data (full circles) and [11] (open circles) are plotted.

Several interesting features should be mentioned:

- The kinematic limit extends far below $x_{F}=-1$.

- There is no indication of diffractive peak at $x_{F} \sim-1$.

- The maximum of the distributions in backward region is located at $x_{F} \sim-0.92$ rather than $x_{F}=-1$.

The $p_{T}$ integrated $d n / d x_{F}$ distributions for protons and neutrons are presented in Fig. 7 in the projectile hemisphere together with the neutron results from $p+p$ collisions (dotted lines). Protons and neutrons show within errors the same transfer towards the center when passing from $\mathrm{p}+\mathrm{p}$ to $\mathrm{p}+\mathrm{C}$ reaction. The densities are equal at $x_{F} \sim 0.3$. Densities from $\mathrm{p}+\mathrm{C}$ are bigger at lower $x_{F}$, reaching increase of about 1.3 at $x_{F}=0.1$, and smaller at larger $x_{F}$ decreasing to 0.6 of the $\mathrm{p}+\mathrm{p}$ densities at $x_{F}$ towards +1 . The latter value corresponds to the expected fraction of single projectile collisions in $\mathrm{p}+\mathrm{C}$ interactions derived from the nuclear density distribution and from the inelastic cross sections [5]. 


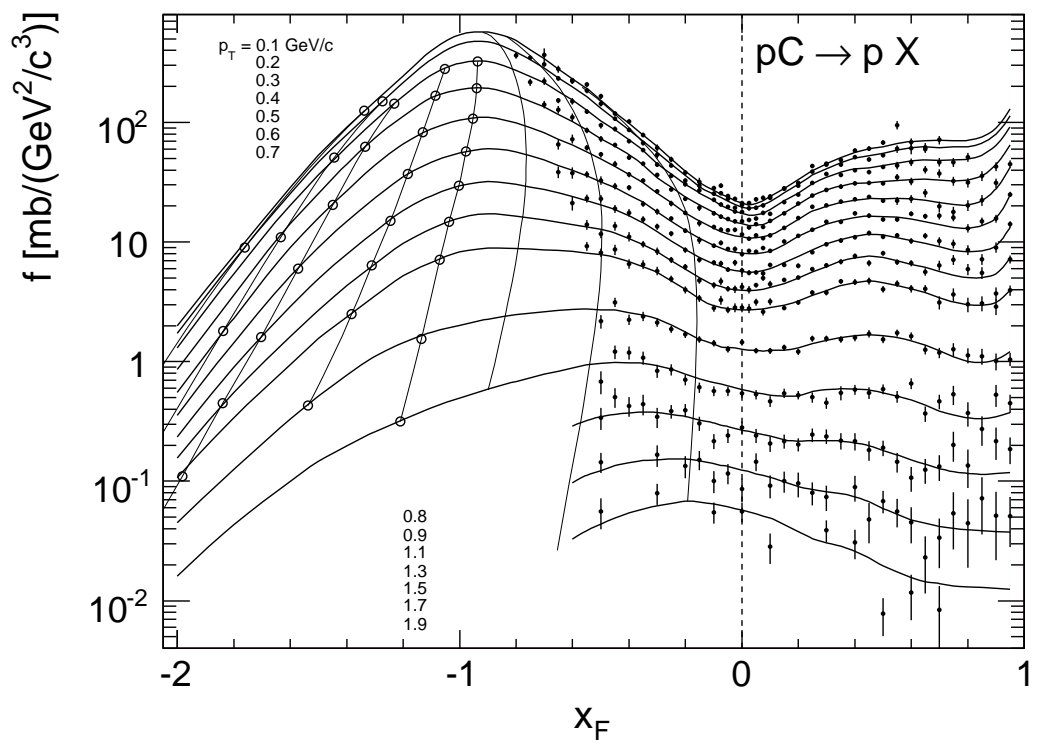

Figure 6. Invariant cross sections at fixed $p_{T}$ as a function of $x_{F}$. Full circles: NA49 data, open circles: data from [11]. The thin lines show the cross section at fixed angles of $10^{\circ}, 30^{\circ}$ and $50^{\circ}$.

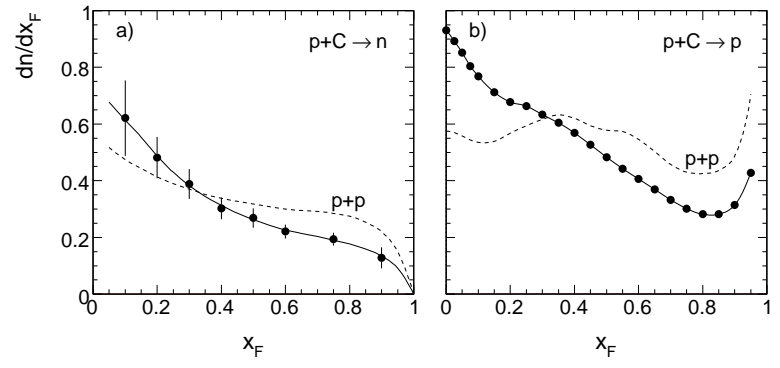

Figure 7. The $p_{T}$ integrated neutron and proton density distribution $d n / d x_{F}$ in $\mathrm{p}+\mathrm{C}$ (full circles) and in $\mathrm{p}+\mathrm{p}$ (dotted line) collisions.

\subsection{Deuterons and tritons}

Deuterons and tritons can be identified via specific energy loss measurement by the NA49 detector at lab momenta below $2 \mathrm{GeV} / \mathrm{c}$ for deuterons and $3 \mathrm{GeV}$ for tritons. The density ratio of deuterons to protons $R_{d}$ and tritons to protons $R_{t}$ are shown in Fig. 5.2 as a function of $x_{F}$, where the transformation from lab to $\mathrm{cms}$ system is performed assuming the proton mass. In the figure are also presented the results from [12] (open symbols) which show good agreement as in the case of the proton data [11]. The ratio $R_{t}$ is suppressed with respect to $R_{d}$ by a factor of 0.1 to 0.2 depending on $p_{T}$ but independent on $x_{F}$. These ratios are for the first time available at low $p_{T}$ and in the approach to $x_{F}=0$, where $R_{d}$ decreases to values of about $1 \%$.

\subsection{Three component mechanism}

The final state of $\mathrm{p}+\mathrm{A}$ collisions consists of three basic components:

- The fragmentation of the projectile particle

- The fragmentation of the target nucleons

- The intra-nuclear cascading, generated by the interaction of the participating nucleons and secondary produced hadrons inside the nucleus.
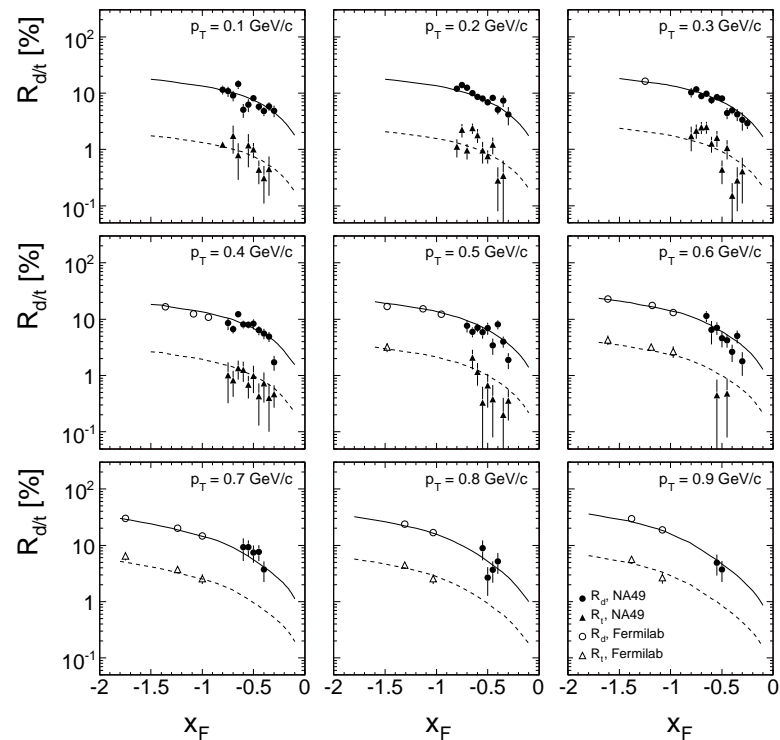

Figure 8. Deuteron and triton to proton density ratios $R_{d}$ and $R_{t}$ as a function of $x_{F}$ for fixed values of $p_{T}$ between 0.1 and $0.9 \mathrm{GeV} / \mathrm{c}$. The full lines give the two-dimensional data interpolation established for $R_{d}$, the broken lines are the same multiplied by the suppression factors, assumed $x_{F}$ independent, see [6].

These components have been separated and quantified for pion production $[5,7]$ and baryon production [6] in $\mathrm{p}+\mathrm{C}$ collisions. An example of the net proton $p_{T}$ integrated $d n / d x_{F}$ distribution is shown in Fig. 9 where the total yield and the three components are indicated with different lines.

It has to be noted that this separation relies essentially on experimental input. There is only one assumption made concerning the target component and no other free parameters are used in the analysis. The target component is predicted from $\mathrm{p}+\mathrm{p}$ collisions, assuming that the hadronization of the hit target nucleus is equivalent to the hadronization of the elementary nucleon-nucleon interactions, taking into account the isospin symmetry and multiplying by the mean number of collisions $\langle v\rangle$ that the projectile suf- 


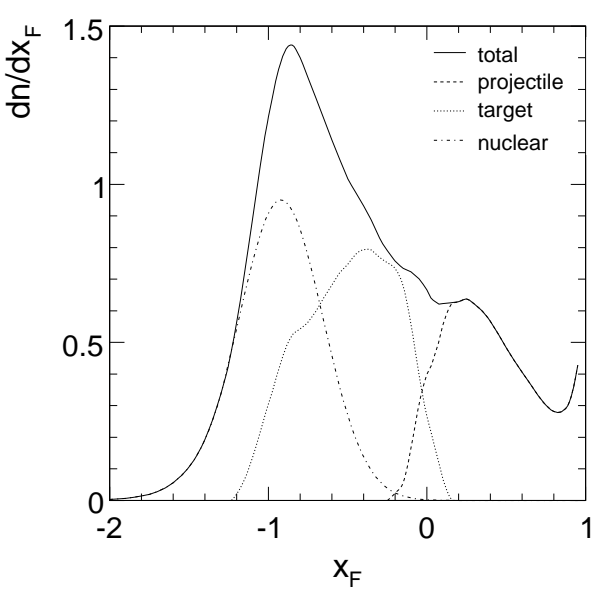

Figure 9. The nuclear, target and projectile components for protons in $\mathrm{p}+\mathrm{C}$ collisions.

fers on its way through the target nucleus, see [6]. For the $\mathrm{p}+\mathrm{C}$ interaction $\langle v\rangle$ is equal to 1.6 , see [4]. The projectile and nuclear components follow, without additional assumptions, from the subtraction of the target fragmentation from the measured total yield.

\section{Survey of backward production of protons and pions in $\mathrm{p}+\mathrm{C}$ interactions}

In Sect. 5.1, Fig. 6, it was shown that backward proton production is peaked at around $x_{F}=-0.92$ and not at $x_{F}=-1$. One mechanism for this baryon transfer can be pion production in intra-nuclear cascading [2]. To study this phenomenon a survey of backward production of protons and pions in $\mathrm{p}+\mathrm{C}$ collisions has been performed [7]. This survey covers the region of $\Theta_{\text {lab }}$ from 10 to 180 degrees, $p_{\text {lab }}$ from 0.2 to $1.2 \mathrm{GeV} / \mathrm{c}$ and beam momentum $p_{\text {beam }}$ from 1 to $400 \mathrm{GeV} / \mathrm{c}$. About 3500 data points coming from 19 experiments have been localized [7]. As these experiments use in general different variables and binning, all results were transformed to invariant cross sections (1) and expressed in the three variables $\Theta_{\text {lab }}, p_{\text {lab }}$ and $1 / \sqrt{s}$. A three dimensional interpolation in these three variables was performed which uses the physics constraints of smoothness, continuity and regularity at the phase space boundaries, for details see [7]. An example of $\pi^{-}$distributions as a function of $1 / \sqrt{s}$ at fixed $p_{\text {lab }}$ and $\Theta_{\text {lab }}=67$ degrees together with the global data interpolation is presented in Fig. 10.

The data interpolation establishes a strong constraint for possible experimental deviations. In fact 4 of the 19 data sets have been shown to be inconsistent with all other results.

\section{Conclusions}

New inclusive data on kaon and baryon production in $\mathrm{p}+\mathrm{p}$ interactions and baryon and light ion production in $\mathrm{p}+\mathrm{C}$ collisions at $158 \mathrm{GeV} / \mathrm{c}$ beam momentum have been presented. These results are part of the systematic study of soft hadronic interactions by the NA49 experiment, combining wide phase space coverage and systematic errors below $5 \%$.

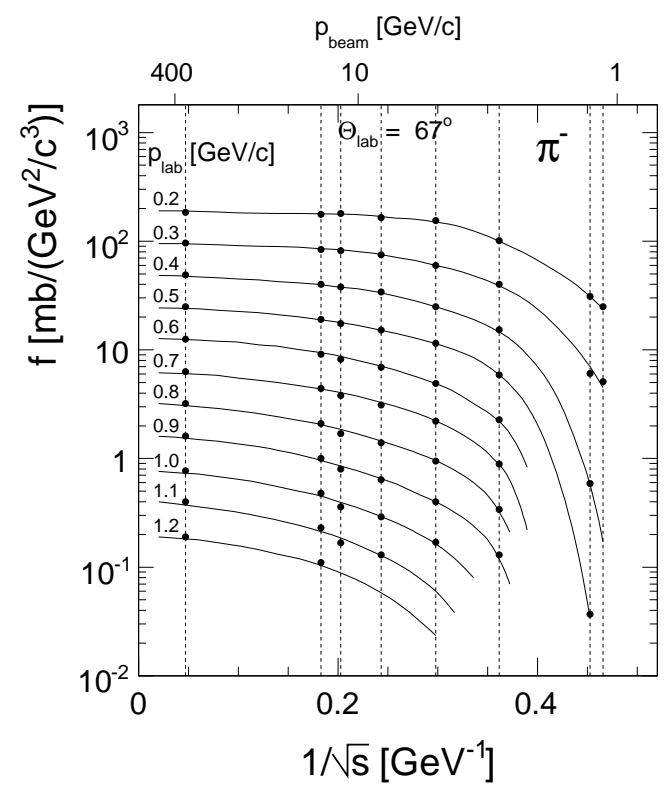

Figure 10. Invariant cross sections for $\pi^{-}$in $\mathrm{p}+\mathrm{C}$ collisions as a function of $1 / \sqrt{s}$ at fixed $p_{\text {lab }}$ and $\Theta_{\text {lab }}=67$ degrees. The solid lines represent the global data interpolation.

The comparison of the results on proton and neutron production in $\mathrm{p}+\mathrm{p}$ interactions and deep inelastic $\mathrm{e}+\mathrm{p}$ collisions at HERA reveals hadronic factorization in the target fragmentation region.

New evaluation of kaon yields in $\mathrm{p}+\mathrm{p}$ collisions has been established in the range $3<\sqrt{s}<1800 \mathrm{GeV}$ where the relation $\mathrm{K}_{S}^{0}=0.5\left(\mathrm{~K}^{+}+\mathrm{K}^{-}\right)$is shown to be valid for $\sqrt{s}>5 \mathrm{GeV}$.

A survey of backward production of protons and pions in the range of lab angles from 10 to 180 degrees, from 0.2 to $1.2 \mathrm{GeV} / \mathrm{c}$ in lab momentum and from 1 to $400 \mathrm{GeV} / \mathrm{c}$ in projectile momentum has been performed. Over 3500 data points from 19 experiments have been used in order to establish a consistent description of proton and pion production in the backward hemisphere.

\section{Acknowledgements}

This work was supported by the Bulgarian National Science Fund (Ph-09/05).

\section{References}

[1] C. Alt et al., Eur. Phys. J. C45 (2006) 343

[2] T. Anticic et al., Eur. Phys. J. C65 (2010) 9

[3] T. Anticic et al., Eur. Phys. J. C68 (2010) 1

[4] C. Alt et al., Eur. Phys. J. C49 (2007) 897

[5] G. Barr et al., Eur. Phys. J. C49 (2007) 919

[6] B. Baatar et al., arXiv:1207.6520v1[hep-ex]

[7] O. Chvala et al., arXiv:1210.6775[nucl-ex]

[8] S. Chekanov et al., Nucl. Phys. B658 (2003) 3

[9] S. Chekanov et al., Nucl. Phys. B776 (2007) 1

[10] A. M. Rossi et al., Nucl. Phys. B84 (1975) 269

[11] Y. D. Bayukov et al., Phys. Rev. C20, (1979) 764

[12] S. Frankel et al., Phys. Rev. C20, (1979) 2257 